\title{
Clinical Pharmacology of Fast-Acting Insulin Aspart Versus Insulin Aspart Measured as Free or Total Insulin Aspart and the Relation to Anti-Insulin Aspart Antibody Levels in Subjects with Type 1 Diabetes Mellitus
}

\author{
Hanne Haahr ${ }^{1} \cdot$ Thomas R. Pieber $^{2}$ - Chantal Mathieu ${ }^{3} \cdot$ Theis Gondolf $^{1} \cdot$ Masanari Shiramoto $^{4} \cdot$ Lars Erichsen $^{1}$. \\ Tim Heise ${ }^{5}$
}

Published online: 7 November 2018

(c) The Author(s) 2018

\begin{abstract}
Background Fast-acting insulin aspart (faster aspart) is an ultra-fast-acting formulation of insulin aspart (IAsp). This post hoc analysis investigated the pharmacokinetics of faster aspart versus IAsp, measured as free or total IAsp, and the relationship between anti-IAsp antibodies and the pharmacokinetics/pharmacodynamics of faster aspart and IAsp.

Methods Free and total IAsp concentrations and anti-IAsp antibodies were determined in adults with type 1 diabetes mellitus receiving subcutaneous faster aspart and/or IAsp in four single-dose clinical pharmacology trials $(n=175)$ and a 26-week phase IIIa trial $(n=1040)$. Pharmacodynamics were assessed by euglycaemic clamp or meal test, respectively.

Results The pharmacokinetic profile was left-shifted and early exposure was greater with faster aspart versus IAsp independent of free or total IAsp assay. The faster aspart-IAsp difference in the time to 50\% of maximum IAsp concentration in the early part of the pharmacokinetic profile $\left(t_{\text {Early }} 50 \%\right.$ Cmax $)$ [95\% confidence interval (CI)] was $-8.8[-10.0$ to -7.5$]$ and -7.6 [ -8.8 to -6.4$] \mathrm{min}$ for free and total IAsp, respectively. The faster aspart/IAsp ratio for the area under the concentration-time curve (AUC) for IAsp from time zero to $30 \mathrm{~min}\left(\mathrm{AUC}_{\mathrm{IAsp}, 0-30 \mathrm{~min}}\right)$ [95\% CI] was 1.88 [1.74-2.04] and 1.77 [1.64-1.90] for free and total IAsp. Higher anti-IAsp antibody levels were associated with a lower ratio of free/total IAsp for the total AUC for IAsp $\left(\mathrm{AUC}_{\mathrm{IAsp}, 0-t}\right)$. Early glucose-lowering effect (AUC for the glucose infusion rate [GIR] from time zero to $60 \mathrm{~min}$ [AUC $\mathrm{AIIR}, 0-60 \mathrm{~min}]$ ) was greater by $25-44 \%$ for faster aspart versus IAsp independent of anti-IAsp antibody levels. Total glucose-lowering effect (total AUC for GIR [AUC $\left.{ }_{\mathrm{GIR}, 0-t}\right]$ ) in a clamp and 1-h postprandial glucose increment in a meal test appeared essentially unaffected by anti-IAsp antibodies.

Conclusions Faster aspart provides accelerated pharmacokinetics versus IAsp regardless if based on free or total IAsp assay. Higher anti-IAsp antibodies increase total IAsp concentrations but do not influence faster aspart nor IAsp pharmacodynamics. ClinicalTrials.gov identifiers NCT01618188, NCT02003677, NCT01934712, NCT02568280, NCT01831765.
\end{abstract}

Electronic supplementary material The online version of this article (https://doi.org/10.1007/s40262-018-0718-6) contains supplementary material, which is available to authorized users.

Hanne Haahr

hhaa@novonordisk.com

1 Novo Nordisk A/S, Vandtårnsvej 114, 2860 Søborg, Denmark

2 Department of Internal Medicine, Medical University of Graz, Auenbruggerplatz 15, 8036 Graz, Austria
3 Clinical and Experimental Endocrinology, University Hospital Leuven, KU Leuven, UZ Herestraat 49, Box 902, 3000 Leuven, Belgium

4 SOUSEIKAI, Hakata Clinic, 6-18 Tenyamachi, Hakata-ku, Fukuoka 812-0025, Japan

5 Profil, Hellersbergstrasse 9, 41460 Neuss, Germany 


\section{Key Points}

In a post hoc analysis of four clinical pharmacology trials in subjects with type 1 diabetes mellitus, it was shown that faster aspart provides accelerated pharmacokinetics compared with insulin aspart (IAsp) independent of whether free or total insulin is assayed.

Higher anti-IAsp antibody levels were associated with greater exposure for total versus free insulin-both in the clinical pharmacology trials and based on sparse pharmacokinetic sampling in a phase IIIa trial with faster aspart and IAsp.

Presence of anti-IAsp antibodies does not appear to affect the greater early glucose-lowering effect provided by faster aspart versus IAsp, nor does the level of antiIAsp antibodies appear to influence the overall glucoselowering effect of faster aspart and IAsp.
IAsp [16]. However, it may also be relevant and has been requested by some regulatory authorities to compare the pharmacokinetics of faster aspart and IAsp based on total IAsp measurements. Such an investigation was possible because both free and total IAsp were measured in selected trials in the faster aspart clinical development programme.

Thus, the main purpose of the current investigation was to compare the pharmacokinetics between faster aspart and IAsp based on both total and free IAsp measurements in subjects with T1DM. This was done in a post hoc analysis of the four clinical pharmacology trials in the faster aspart clinical development programme where both free and total IAsp measurements were available. Furthermore, the influence of anti-IAsp antibody levels on the exposure and glucoselowering effect of faster aspart and IAsp was explored. The analysis was supplemented with pharmacokinetic and meal test results from a large phase IIIa trial with faster aspart and IAsp.

\section{Methods}

\section{Introduction}

In insulin-treated patients with diabetes, insulin may exist in the circulation both as bound and free insulin. The bound fraction is due to reversible binding to endogenous antiinsulin antibodies [1, 2]. Recently, anti-insulin antibodies have received less attention because of reduced impurities of insulin products and widespread use of human insulin and human-derived insulin analogues. Still, therapeutic insulins continue to be immunogenic in many patients [3-5]. Consequently, interference by anti-insulin antibodies in insulin assays remains an important issue [6]. In contrast to direct measurement of total insulin (bound plus unbound), determination of free insulin concentrations requires prior removal of anti-insulin antibodies before analysis, a step accomplished by polyethylene glycol precipitation $[1,2]$. In the past, pharmacokinetic characterisation of exogenous insulin products in previously insulin-treated subjects was based on measurement of either total insulin $[7,8]$ or free insulin [9-11].

Fast-acting insulin aspart (faster aspart) has been developed as an ultra-fast-acting formulation of the mealtime insulin, insulin aspart (IAsp) [12, 13]. Faster aspart contains two additional excipients, L-arginine and niacinamide, to facilitate formulation stability and faster initial absorption $[14,15]$. Importantly, the IAsp molecule in faster aspart is unmodified compared with the original IAsp formulation. Based on free IAsp measurements in subjects with type 1 diabetes mellitus (T1DM), faster aspart provides $\sim 5 \mathrm{~min}$ earlier onset of appearance and twice-as-high early insulin exposure in the first 30 min post-dosing compared with

\subsection{Study Design}

The current post hoc analysis included four clinical pharmacology trials contributing pharmacokinetic and anti-IAsp antibody data (Trials 1-4) (Electronic Supplementary Material [ESM] Online Resource 1, Table S1). Three of these trials used a glucose clamp and therefore also contributed pharmacodynamic data (Trials 1-3). All four clinical pharmacology trials were randomised, double-blind, crossover trials and were conducted at Profil, Neuss, Germany (Trials 1 and 2) [15, 17], SOUSEIKAI, Hakata Clinic, Fukuoka, Japan (Trial 3) [18] and Department of Internal Medicine, Medical University of Graz, Austria (Trial 4) [19]. Independent of trial centre, the clinical pharmacology trials used comparable overall methodology and had a similar design including IAsp as comparator. It was therefore assessed valid to pool the four trials in a combined analysis. For the investigation of the association between anti-IAsp antibody levels and exposure as well as the glucose-lowering effect of faster aspart and IAsp, the clinical pharmacology data were supplemented with data from a phase IIIa multicentre, active-controlled, randomised, parallel-group trial with faster aspart and IAsp in subjects with T1DM (Trial 5) [20].

In each trial, health authorities and local ethics committees reviewed and approved the protocol, protocol amendments, consent form and subject information sheets according to local regulations prior to trial initiation. All trials were performed in accordance with the Declaration of Helsinki and Good Clinical Practice. Participants provided written informed consent before initiation of any trial-related activities. The trials were registered at 
ClinicalTrials.gov: NCT01618188 (Trial 1), NCT02003677

(Trial 2), NCT01934712 (Trial 3), NCT02568280 (Trial 4) and NCT01831765 (Trial 5).

\subsection{Study Populations}

The combined analysis of clinical pharmacology trials was based on data from 175 adults with T1DM aged $\geq 18$ years. The analysis of data from the phase IIIa trial included 1040 adults with T1DM aged $\geq 18$ years. For all trials, only subjects with available pharmacokinetic data for both free and total IAsp as well as data on anti-IAsp antibodies were included in the current analysis.

Key inclusion criteria for each trial are shown in ESM Online Resource 1, Table S1. In the clinical pharmacology trials, subjects had received treatment with multiple daily insulin injections or insulin pump therapy for $\geq 12$ months with total insulin dose $<1.2$ (I) $\mathrm{U} / \mathrm{kg} /$ day and bolus insulin dose $<0.7$ (I) $\mathrm{U} / \mathrm{kg} /$ day. In the phase IIIa trial, subjects had been treated in a basal-bolus insulin treatment regimen for $\geq 12$ months and with insulin detemir or insulin glargine as basal insulin for $\geq 4$ months. General exclusion criteria in the clinical pharmacology trials included clinically significant concomitant diseases (malignant neoplasms or cardiovascular, renal, hepatic, respiratory, gastrointestinal or haematological diseases), history of severe allergies to medication or foods or a history of severe anaphylactic reaction, smoking (not in Trial 4) or current treatment with drug(s) that might interfere with glucose metabolism. Exclusion criteria in the phase IIIa trial included any use of anti-diabetes drugs other than insulin $\leq 3$ months prior to screening, known or suspected hypersensitivity to any of the trial products or related products, recurrent severe hypoglycaemia, hypoglycaemic unawareness as judged by the investigator or hospitalisation for diabetic ketoacidosis $\leq 6$ months prior to screening. Pregnant or breastfeeding women were excluded from all trials.

\subsection{Study Procedures and Assessments}

In all four clinical pharmacology trials, subjects received single subcutaneous dosing of faster aspart $(100 \mathrm{U} / \mathrm{mL}$; Novo Nordisk, Bagsværd, Denmark) and IAsp (NovoRapid ${ }^{\circledR}$; $100 \mathrm{U} / \mathrm{mL}$; Novo Nordisk) into a lifted skin fold of the lower abdominal wall above the inguinal area. In Trial 1, subjects received two formulations of faster aspart, but the current analysis included only data for the formulation pursued in further clinical development. In Trials 1-3, the dose was fixed at $0.2 \mathrm{U} / \mathrm{kg}$, while subjects in Trial 4 received an individualised dose of $0.06-0.28 \mathrm{U} / \mathrm{kg}$ (identical at both dosing visits for each subject) based on the subject's customary insulin:carbohydrate ratio. The two dosing visits were separated by at least 3 days' washout, where subjects resumed their usual insulin treatment. Blood samples for serum IAsp measurement were drawn at pre-specified timepoints shown in ESM Online Resource 1, Table S2.

In Trials 1, 2 and 3, which contributed pharmacodynamic data, an automated euglycaemic glucose clamp (Biostator ${ }^{\circledR}$, MTB Medizintechnik, Amstetten, Germany in Trial 1; ClampArt $^{\circledR}$, Profil, Neuss, Germany in Trial 2; STG-22 Artificial Endocrine Pancreas, NIKKISO Co. Ltd., Tokyo, Japan in Trial 3) with a target blood glucose concentration of $5.5 \mathrm{mmol} / \mathrm{L}(100 \mathrm{mg} / \mathrm{dL})$ was performed up to $12 \mathrm{~h}$ post-dosing. Clamp procedure details have been provided previously $[15,18]$. In Trial 4, a 6-h mixed meal test was conducted and postprandial glucose turnover was assessed by the triple-tracer method (data published previously [19]).

In the clinical pharmacology trials, the subjects' current insulin therapy was washed out before each trial product administration. Subjects were not allowed to take insulin degludec $\leq 72 \mathrm{~h}$ pre-dose, insulin glargine or insulin detemir $\leq 48 \mathrm{~h}$ pre-dose, intermediate-acting insulin (e.g. neutral protamine Hagedorn [NPH] insulin) $\leq 22 \mathrm{~h}$ pre-dose or IAsp $\leq 12 \mathrm{~h}$ (for bolus) or $\leq 8 \mathrm{~h}$ (for basal infusion using a pump) pre-dose.

In the phase IIIa trial, subjects were randomised to receive 26 weeks' treatment with double-blind mealtime faster aspart, mealtime IAsp or open-label post-meal faster aspart in a basal-bolus regimen with insulin detemir as basal insulin. Mealtime bolus insulin was injected $0-2$ min premeal, while post-meal faster aspart was injected $20 \mathrm{~min}$ after the start of a meal. At 26 weeks of treatment, in connection with a standardised liquid meal test, a fixed dose of $0.1 \mathrm{U} /$ $\mathrm{kg}$ was administered 0-2 min pre-meal (mealtime faster aspart and IAsp) or $20 \mathrm{~min}$ after the start of a meal (postmeal faster aspart). Blood sampling for anti-IAsp antibody measurement was done before the meal test, while blood sampling for pharmacokinetic assessment was done at 1 and $2 \mathrm{~h}$ after the start of meal ingestion. Plasma glucose was measured immediately pre-meal and every hour until $4 \mathrm{~h}$ post-meal. Subjects were required to attend the meal test fasting and abstain from any insulin injection, and medication to be taken during or after a meal, until the meal test was completed.

Free and total serum IAsp concentrations were measured using a validated IAsp-specific enzyme-linked immunosorbent assay with a lower limit of quantification of $10 \mathrm{pmol} / \mathrm{L}$. Before measurement of free IAsp, serum samples were precipitated using polyethylene glycol to obtain a supernatant fraction containing free IAsp.

Total anti-IAsp antibodies (IAsp-specific antibodies as well as those cross-reacting with human insulin) were measured by a validated subtraction radioimmunoassay using ${ }^{125}$ I-labelled IAsp. Serum samples were incubated with ${ }^{125}$ I-labelled IAsp in the presence or absence of unlabelled antigen (IAsp or human insulin). Radiolabelled IAsp 
bound to antibodies in the sample was precipitated, measured and expressed as percent bound radioactivity (B) of the total amount of radioactivity (T) added to the sample. Thus, the $\% \mathrm{~B} / \mathrm{T}$ value is proportional to the amount of antiIAsp antibody present in the sample. Since the assay is a subtraction radioimmunoassay, where the assay background is subtracted, $\% \mathrm{~B} / \mathrm{T}$ varies around zero for antibody-negative samples due to assay variation. Thus, $\% \mathrm{~B} / \mathrm{T}$ may be slightly below zero for antibody-negative samples.

\subsection{Study Endpoints}

In the clinical pharmacology trials, onset of exposure was assessed by the endpoints onset of appearance, time to $50 \%$ of maximum IAsp concentration in the early part of the pharmacokinetic profile ( $t_{\text {Early } 50 \% C \max }$ ) and time to maximum IAsp concentration $\left(t_{\max }\right)$. Early IAsp exposure was evaluated by the areas under the concentration-time curve (AUCs) for serum IAsp from time zero to 15 ( $\left.\mathrm{AUC}_{\mathrm{IAsp}, 0-15 \mathrm{~min}}\right), 30$ $\left(\mathrm{AUC}_{\mathrm{IAsp}, 0-30 \mathrm{~min}}\right)$ and $60 \mathrm{~min}\left(\mathrm{AUC}_{\mathrm{IAsp}, 0-60 \mathrm{~min}}\right)$, respectively. Offset of IAsp exposure was assessed by the time to $50 \%$ of maximum IAsp concentration in the late part of the pharmacokinetic profile $\left(t_{\text {Late } 50 \% \text { Cmax }}\right)$ and the AUC for serum IAsp from $2 \mathrm{~h}\left(\mathrm{AUC}_{\mathrm{IAsp}, 2-t}\right)$. Overall IAsp exposure was evaluated by total AUC for serum IAsp $\left(\mathrm{AUC}_{\mathrm{IAsp}, 0-t}\right)$ and maximum IAsp concentration ( $C_{\text {max,IAsp }}$ ). Early glucose-lowering effect was assessed by the AUC for the glucose infusion rate (GIR) from time zero to $60 \mathrm{~min}\left(\mathrm{AUC}_{\mathrm{GIR}, 0-60 \mathrm{~min}}\right)$, offset of glucoselowering effect was evaluated by the AUC for the GIR from $2 \mathrm{~h}\left(\mathrm{AUC}_{\mathrm{GIR}, 2-t}\right)$ and overall glucose-lowering effect was assessed by the total GIR (total AUC for GIR [AUC $\left.{ }_{\mathrm{GIR}, 0-t}\right]$ ). All endpoints were calculated as previously described [16].

In the phase IIIa trial (Trial 5), $\mathrm{AUC}_{\mathrm{IAsp}, 0-t}$ was predicted from the two IAsp concentration measurements performed post-dose at 26 weeks of treatment. The prediction was based on data from available clinical pharmacology trials with faster aspart and IAsp (described in ESM Online Resource 1). Moreover, the 1-h postprandial plasma glucose increment $\left(\Delta \mathrm{PPG}_{1 \mathrm{~h}}\right)$ of the meal test was calculated.

\subsection{Statistical Analyses}

All statistical analyses were performed using $\mathrm{SAS}^{\circledR}$ version 9.4 (SAS Institute, Cary, NC, USA). Pharmacokinetic endpoints from the clinical pharmacology trials were compared between faster aspart and IAsp in a linear mixed model with treatment and trial as fixed effects and subject as random effect. Onset of appearance, $t_{\text {Early } 50 \% C \max }, t_{\max }$ and $t_{\text {Late }} 50 \% \mathrm{Cmax}$ were analysed on an absolute scale: least square means for each treatment as well as treatment differences and $95 \%$ confidence intervals (CIs) were estimated. All AUCs for serum IAsp and $C_{\text {max,IAsp }}$ were log-transformed before analysis, and least square means for each treatment as well as treatment ratios and $95 \%$ CIs were estimated.

The potential effect of anti-IAsp antibodies on the exposure of free and total IAsp was investigated by plotting the relationship between the level of anti-IAsp antibodies and the ratio of measured exposure of free versus total IAsp. Plots were prepared for the combined clinical pharmacology trials and for the phase IIIa trial. Only those subjects with available data for anti-IAsp antibody level and $\mathrm{AUC}_{\mathrm{IAsp}, 0-t}$ for both free and total IAsp (for faster aspart and/or IAsp) were included in the plot.

Based on the clinical pharmacology trials, the potential effect of anti-IAsp antibodies on the early glucose-lowering effect and on the offset of glucose-lowering effect of faster aspart versus IAsp was investigated by calculating the mean ratio of faster aspart/IAsp for $\mathrm{AUC}_{\mathrm{GIR}, 0-60 \mathrm{~min}}$ and $\mathrm{AUC}_{\mathrm{GIR}, 2-t}$ for each of four subgroups ranging from the lowest to the highest level of anti-IAsp antibodies. In addition, the potential effect of anti-IAsp antibodies on overall glucose-lowering effect of faster aspart and IAsp was investigated by plotting the relationship between level of anti-IAsp antibodies and $\mathrm{AUC}_{\mathrm{GIR}, 0-t}$. Finally, based on the mealtime faster aspart and IAsp treatment arms in the phase IIIa trial, the potential effect of anti-IAsp antibodies on 1-h postprandial glucose increment in a meal test was investigated by plotting the relationship between the level of anti-IAsp antibodies and $\triangle \mathrm{PPG}_{1 \mathrm{~h}}$ for both faster aspart and IAsp.

\section{Results}

\subsection{Subjects}

A total of 175 subjects from the four clinical pharmacology trials were administered both faster aspart and IAsp and contributed to the pharmacokinetic analysis. In 112 subjects from three of the clinical pharmacology trials, pharmacodynamic glucose clamp data were available and included in the pharmacodynamic analysis. Analysis of the phase IIIa trial included the 1040 subjects with available pharmacokinetic data for both free and total IAsp and an anti-IAsp antibody measurement at 26 weeks of treatment. A total of 347, 341 and 352 subjects received mealtime faster aspart, post-meal faster aspart and mealtime IAsp, respectively. Baseline characteristics for both clinical pharmacology trial populations and for the phase IIIa trial population are summarised in ESM Online Resource 1, Table S3. In the pooled population from the clinical pharmacology trials, the majority of subjects were male (65\%), and subjects had a mean age of 42 years (range 21-73 years), body weight of $72.0 \mathrm{~kg}$ (range $48.6-100.0 \mathrm{~kg}$ ), body mass index (BMI) of $23.9 \mathrm{~kg}$ / $\mathrm{m}^{2}$ (range $18.5-28.7 \mathrm{~kg} / \mathrm{m}^{2}$ ), duration of diabetes of 21 years (range 1-57 years), glycosylated haemoglobin $\left(\mathrm{HbA}_{1 \mathrm{c}}\right)$ 
at baseline of $7.3 \%$ (52 mmol/mol) (range 5.2-9.2\%; $30-71 \mathrm{mmol} / \mathrm{mol}$ ) and an anti-IAsp antibody level at baseline of $10.8 \% \mathrm{~B} / \mathrm{T}$ (range -0.1 to $66.9 \% \mathrm{~B} / \mathrm{T}$ ). The subset included in the pharmacodynamic analysis had comparable baseline characteristics. In the phase IIIa trial population, the majority of subjects were male (59\%), and subjects had a mean age of 45 years (range 18-83 years), body weight of $80.0 \mathrm{~kg}$ (range $45.3-140.0 \mathrm{~kg}$ ), BMI of $26.7 \mathrm{~kg} / \mathrm{m}^{2}$ (range $17.0-37.9 \mathrm{~kg} / \mathrm{m}^{2}$ ), duration of diabetes of 20 years (range $1-65$ years $), \mathrm{HbA}_{1 \mathrm{c}}$ at baseline of $7.6 \%(59 \mathrm{mmol} / \mathrm{mol})$ (range $5.6-9.8 \% ; 38-84 \mathrm{mmol} / \mathrm{mol}$ ) and an anti-IAsp antibody level at 26 weeks of treatment of $18.0 \% \mathrm{~B} / \mathrm{T}$ (range $0.2-86.7 \% \mathrm{~B} / \mathrm{T})$.

\subsection{Pharmacokinetics of Faster Aspart Versus Insulin Aspart (IAsp) for Free and Total IAsp}

Comparisons of mean serum IAsp concentration-time curves between faster aspart and IAsp are shown for both free and total IAsp in Fig. 1. As expected, total IAsp concentrations were higher than free IAsp concentrations irrespective of insulin product. But importantly, a similar left-shift of the serum IAsp concentration-time curve was seen with faster aspart versus IAsp independent of whether pharmacokinetics were measured as free or total IAsp.

As seen in Table 1, onset of appearance occurred earlier and $t_{\text {Early } 50 \% C_{\max }}$ and $t_{\max }$ were shorter for faster aspart than IAsp both for free and total IAsp. Furthermore, the quantitative differences between faster aspart and IAsp for onset of appearance, $t_{\text {Early }} 50 \% C_{\max }$ and $t_{\max }$ were comparable for free and total IAsp (Table 1 and ESM Online Resource 1, Table S4).

Greater early exposure up to 60 min after dosing was seen with faster aspart than with IAsp irrespective of pharmacokinetic assay method, and the quantitative differences between faster aspart and IAsp for early exposure were in the same range for free and total IAsp (Table 2 and ESM Online Resource 1, Table S5). For example, $\mathrm{AUC}_{\mathrm{IAsp}, 0-30 \mathrm{~min}}$ was close to twice-as-great with faster aspart versus IAsp for both free and total IAsp (treatment ratios for faster aspart/ IAsp of 1.88 and 1.77 , respectively).

The offset of IAsp exposure occurred earlier for faster aspart than for IAsp independent of assay method. Moreover, the relative differences between faster aspart and IAsp for $t_{\text {Late } 50 \% C \max }$ and $\mathrm{AUC}_{\text {IAsp,2- } t}$ were comparable for free and total IAsp (Table 3 and ESM Online Resource 1, Table S6).

Total exposure $\left(\mathrm{AUC}_{\mathrm{IAsp}, 0-t}\right)$ and $C_{\text {max,IAsp }}$ were comparable between faster aspart and IAsp both for free IAsp (treatment ratios faster aspart/IAsp [95\% CI] 1.00 [0.97-1.03], $p=0.962 ;$ and 1.04 [0.99-1.09], $p=0.092$, respectively) and for total IAsp (1.01 [0.97-1.04], $p=0.760$; and 1.04
[0.99-1.08], $p=0.101$, respectively) (ESM Online Resource 1, Table S7).

\subsection{Relationship Between Anti-IAsp Antibodies and the Measurement of Free Versus Total IAsp}

The relationship between the level of anti-IAsp antibodies and the ratio of measured exposure of free versus total IAsp is presented in Fig. 2. Independent of trial product (faster aspart or IAsp), there was a clear relation between the antiIAsp antibody level and the ratio of free versus total IAsp for $\mathrm{AUC}_{\mathrm{IAsp}, 0-t}$ both in the clinical pharmacology trials and the phase IIIa trial. Thus, in subjects with low anti-IAsp antibody levels, $\mathrm{AUC}_{\mathrm{IAsp}, 0-t}$ for free IAsp was closer to $\mathrm{AUC}_{\mathrm{IAsp}, 0-t}$ for total IAsp. Conversely, in subjects with high anti-IAsp antibody levels, $\mathrm{AUC}_{\mathrm{IAsp}, 0-t}$ for free IAsp was small relative to $\mathrm{AUC}_{\mathrm{IAsp}, 0-t}$ for total IAsp.

\subsection{Pharmacodynamics of Faster Aspart Versus IAsp in Relation to Anti-IAsp Antibody Level}

In the four quartiles of anti-IAsp antibody levels in the pooled analysis of clinical pharmacology trials, early glucose-lowering effect $\left(\mathrm{AUC}_{\mathrm{GIR}, 0-60 \text { min }}\right)$ was greater with faster aspart than with IAsp and late glucose-lowering effect $\left(\mathrm{AUC}_{\mathrm{GIR}, 2-t}\right)$ was smaller with faster aspart than with IAsp (Table 4). Furthermore, there was essentially no relationship between the level of anti-IAsp antibodies and total glucoselowering effect in a clamp setting (Fig. 3a) or between the level of anti-IAsp antibodies and 1-h postprandial glucose increment in a meal test (Fig. 3b).

\section{Discussion}

The main finding of the present study was the consistently faster absorption, greater early exposure and earlier offset of exposure for faster aspart than IAsp when using either free or total IAsp to determine the pharmacokinetic properties in subjects with T1DM. Although the general exposure level for both insulin products was almost twice as high for total versus free IAsp, the onset of appearance, $t_{\text {Early }} 50 \%$ Cmax and $t_{\max }$ were remarkably similar for free and total IAsp. Moreover, the quantitative differences between faster aspart and IAsp for early exposure were in the same range for free and total IAsp, and the relative differences between faster aspart and IAsp for offset of exposure were comparable for the free and total IAsp assay method. It was evident from the present findings that the level of anti-IAsp antibodies could at least partly explain the different exposure level for free and total IAsp. The ratio of free/total IAsp for $\mathrm{AUC}_{\text {IAsp, } 0-t}$ was shown to decrease with increasing anti-IAsp antibody 
(a)

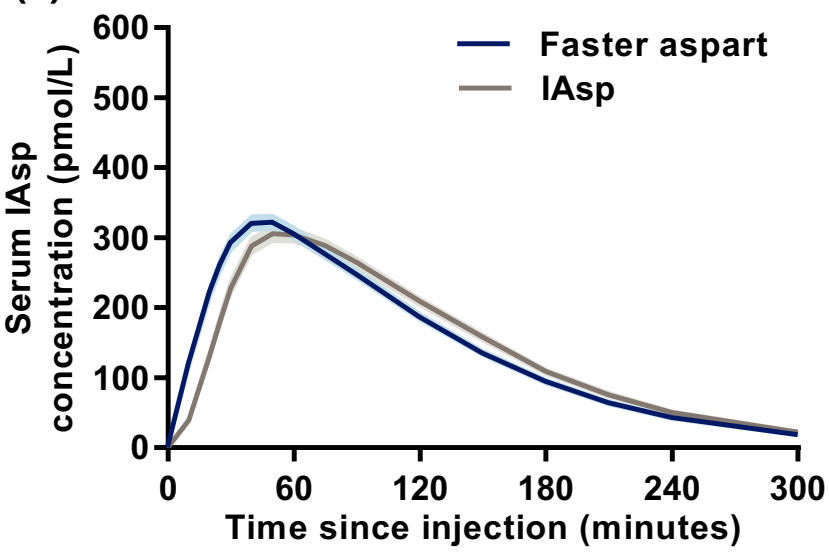

(c)

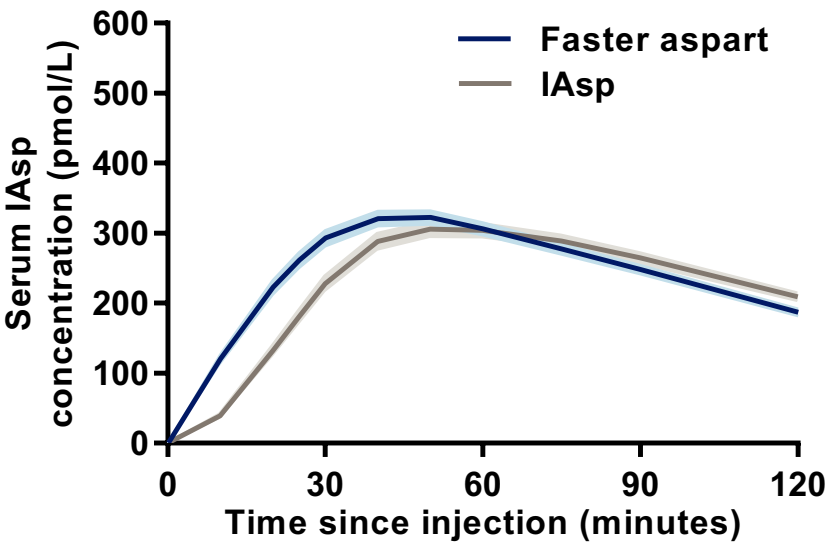

Fig. 1 Mean serum IAsp concentration-time profiles after subcutaneous faster aspart or IAsp administration in subjects with type 1 diabetes mellitus when using an assay to measure free IAsp (a, c) or total IAsp (b, d). 5-h profiles are shown in $\mathbf{a}, \mathbf{b}$, while 2-h profiles are (b)

Total insulin aspart

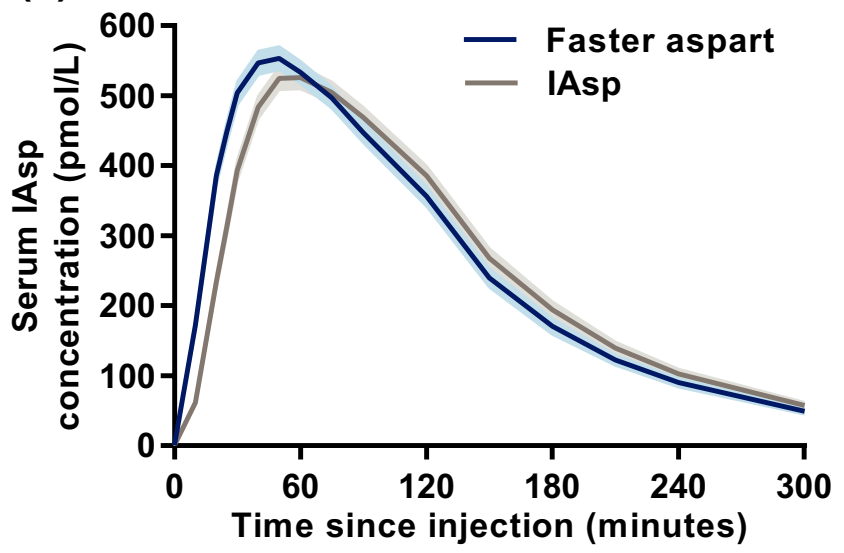

(d)

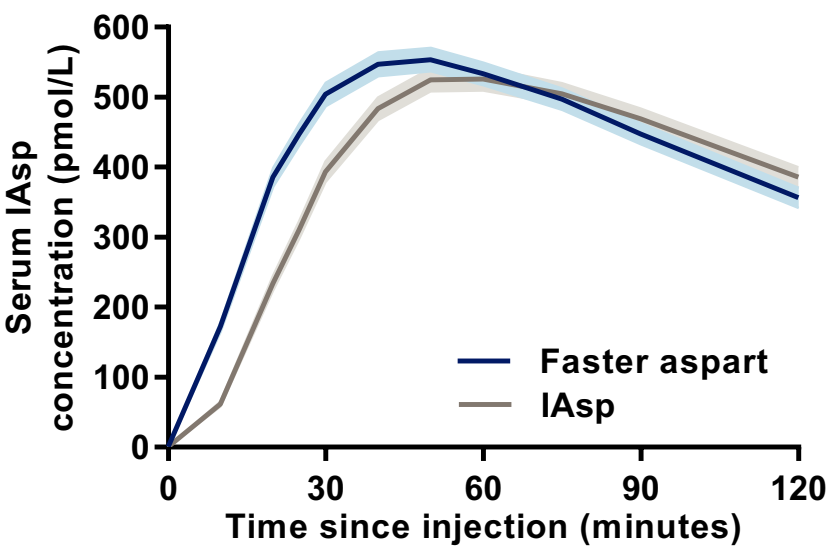

shown in $\mathbf{c}, \mathbf{d}$. Mean pharmacokinetic profiles are based on 175 individual profiles per treatment. The dose was adjusted to $0.2 \mathrm{U} / \mathrm{kg}$ in all subjects. Variability bands show the standard error of the mean. IAsp insulin aspart
Table 1 Onset of exposure for faster aspart versus insulin aspart after subcutaneous administration in subjects with type 1 diabetes mellitus when using an assay to measure free or total insulin aspart

\begin{tabular}{|c|c|c|c|c|}
\hline \multirow[t]{2}{*}{ Variable } & \multicolumn{2}{|l|}{ Free IAsp } & \multicolumn{2}{|l|}{ Total IAsp } \\
\hline & $\begin{array}{l}\text { Treatment difference } \\
\text { (faster aspart - IAsp) } \\
{[95 \% \text { CI] }}\end{array}$ & $p$ value $^{\mathrm{a}}$ & $\begin{array}{l}\text { Treatment difference } \\
\text { (faster aspart - IAsp) } \\
{[95 \% \text { CI }]}\end{array}$ & $p$ value $^{\mathrm{a}}$ \\
\hline Onset of appearance (min) & $-4.1[-4.7$ to -3.6$]$ & $<0.001$ & $-2.5[-2.8$ to -2.1$]$ & $<0.001$ \\
\hline$t_{\text {Early } 50 \% C \max }(\min )^{\mathrm{b}}$ & $-8.8[-10.0$ to -7.5$]$ & $<0.001$ & $-7.6[-8.8$ to -6.4$]$ & $<0.001$ \\
\hline$t_{\max }(\min )$ & $-10.8[-15.4$ to -6.3$]$ & $<0.001$ & $-10.6[-14.7$ to -6.5$]$ & $<0.001$ \\
\hline
\end{tabular}

Results are based on 175 individual pharmacokinetic profiles per treatment

$C I$ confidence interval, IAsp insulin aspart, $t_{\text {Early }} 50 \%$ Cmax time to $50 \%$ of maximum IAsp concentration in the early part of the pharmacokinetic profile, $t_{\max }$ time to maximum IAsp concentration

${ }^{a}$ For treatment comparison of faster aspart vs. IAsp

${ }^{b}$ Not determined in Trial 4 
Table 2 Early exposure for faster aspart versus insulin aspart after subcutaneous administration in subjects with type 1 diabetes mellitus when using an assay to measure free or total insulin aspart

\begin{tabular}{|c|c|c|c|c|}
\hline \multirow[t]{2}{*}{ Variable } & \multicolumn{2}{|l|}{ Free IAsp } & \multicolumn{2}{|l|}{ Total IAsp } \\
\hline & $\begin{array}{l}\text { Treatment ratio } \\
\text { (faster aspart/IAsp) } \\
{[95 \% \mathrm{CI}]}\end{array}$ & $\overline{p \text { value }^{\mathrm{a}}}$ & $\begin{array}{l}\text { Treatment ratio } \\
\text { (faster aspart/IAsp) } \\
{[95 \% \mathrm{CI}]}\end{array}$ & $p$ value $^{\mathrm{a}}$ \\
\hline $\mathrm{AUC}_{\mathrm{IAsp}, 0-15 \min }(\mathrm{pmol} \cdot \mathrm{h} / \mathrm{L})$ & $3.40[3.00-3.85]$ & $<0.001$ & $2.87[2.58-3.20]$ & $<0.001$ \\
\hline $\mathrm{AUC}_{\mathrm{IAsp}, 0-30 \min }(\mathrm{pmol} \cdot \mathrm{h} / \mathrm{L})$ & $1.88[1.74-2.04]$ & $<0.001$ & $1.77[1.64-1.90]$ & $<0.001$ \\
\hline $\mathrm{AUC}_{\mathrm{IAsp}, 0-60 \min }(\mathrm{pmol} \cdot \mathrm{h} / \mathrm{L})$ & $1.29[1.22-1.36]$ & $<0.001$ & $1.26[1.20-1.33]$ & $<0.001$ \\
\hline
\end{tabular}

Results are based on 175 individual pharmacokinetic profiles per treatment. The dose was adjusted to $0.2 \mathrm{U} / \mathrm{kg}$ in all subjects

$A U C_{I A s p, O-\mathrm{x} \text { min }}$ area under the concentration-time curve for IAsp from time zero to $x$ min, $C I$ confidence interval, IAsp insulin aspart

${ }^{\mathrm{a}}$ For treatment comparison of faster aspart vs. IAsp

Table 3 Offset of exposure for faster aspart versus insulin aspart after subcutaneous administration in subjects with type 1 diabetes mellitus when using an assay to measure free or total insulin aspart

\begin{tabular}{|c|c|c|c|c|}
\hline \multirow[t]{2}{*}{ Variable } & \multirow{2}{*}{$\begin{array}{l}\text { Free IAsp } \\
\text { Treatment difference } \\
\text { (faster aspart - IAsp) } \\
{[95 \% \mathrm{CI}]}\end{array}$} & \multirow[b]{2}{*}{$p$ value $^{\mathrm{a}}$} & \multicolumn{2}{|l|}{ Total IAsp } \\
\hline & & & $\begin{array}{l}\text { Treatment difference } \\
\text { (faster aspart - IAsp) } \\
{[95 \% \mathrm{CI}]}\end{array}$ & $p$ value $^{\mathrm{a}}$ \\
\hline$t_{\text {Late } 50 \% C \max }(\mathrm{min})$ & $-13.8[-20.0$ to -7.6$]$ & $<0.001$ & $-14.0[-20.1$ to -7.9$]$ & $<0.001$ \\
\hline \multirow[t]{2}{*}{ Variable } & Free IAsp & & Total IAsp & \\
\hline & $\begin{array}{l}\text { Treatment ratio } \\
\text { (faster aspart/IAsp) } \\
{[95 \% \mathrm{CI}]}\end{array}$ & $p$ value $^{\mathrm{a}}$ & $\begin{array}{l}\text { Treatment ratio } \\
\text { (faster aspart/IAsp) } \\
{[95 \% \mathrm{CI}]}\end{array}$ & $p$ value $^{\mathrm{a}}$ \\
\hline $\mathrm{AUC}_{\mathrm{IAsp}, 2-t}(\mathrm{pmol} \cdot \mathrm{h} / \mathrm{L})$ & $0.88[0.81-0.96]$ & 0.005 & $0.89[0.84-0.96]$ & 0.001 \\
\hline
\end{tabular}

Results are based on 175 individual pharmacokinetic profiles per treatment. For $\mathrm{AUC}_{\mathrm{IAsp}, 2-t}$, the dose was adjusted to $0.2 \mathrm{U} / \mathrm{kg}$ in all subjects $A U C_{I A s p, 2-t}$ area under the concentration-time curve for IAsp from $2 \mathrm{~h}, C I$ confidence interval, IAsp insulin aspart; $t_{\text {Late }} 50 \%$ Cmax time to $50 \%$ of maximum IAsp concentration in the late part of the pharmacokinetic profile

${ }^{a}$ For treatment comparison of faster aspart vs. IAsp

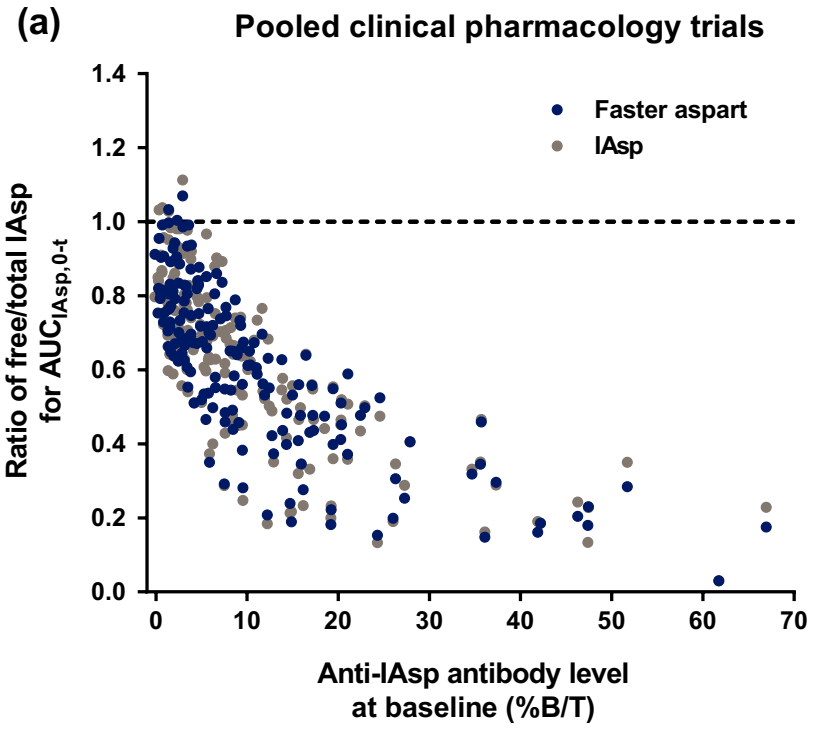

Fig. 2 Relationship between anti-IAsp antibody level and the ratio of free versus total IAsp exposure in four pooled clinical pharmacology trials (a) and a phase IIIa trial (b) with faster aspart and IAsp. The horizontal dotted line represents similarity between free and total

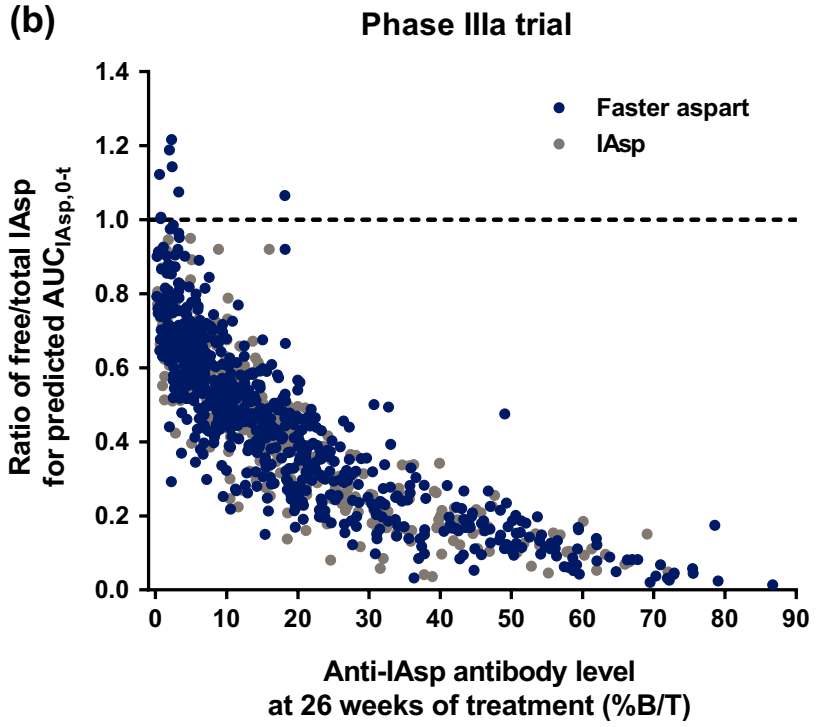

IAsp exposure. Results are based on 175 subjects in the pooled clinical pharmacology trials and 688 (faster aspart) and 352 (IAsp) subjects in the phase IIIa trial. IAsp insulin aspart, $\% B / T$ percent bound/ total 
levels (Fig. 2), which is in accordance with several previous observations with other insulins [6, 21-23].

The consistency between pharmacokinetic results for free and total IAsp with respect to the relative comparison of faster aspart and IAsp may be due to the fact that the active drug substance is the same for both insulins, and therefore the same assay was used to assess pharmacokinetics for both insulin products. Importantly, the current findings cannot necessarily be extended to other insulin products. Thus, it cannot be inferred from the present study that a comparison of pharmacokinetic properties between two different insulins will always lead to similar conclusions irrespective of assaying free or total insulin. Therefore, it is important to obtain consensus on the preferred insulin assay method (free or total insulin), particularly when investigating previously insulin-treated subjects who potentially have developed anti-insulin antibodies. Interestingly, it appears that there is currently a lack of consistency within and between some regulatory authorities with respect to the preferred assay method for assessing exogenous insulin pharmacokinetic properties [12, 13, 24].

There are several examples of hormones other than insulin that are present in the circulation in both a bound and a free fraction, e.g. thyroxine, testosterone, growth hormone

Table 4 Early glucose-lowering effect and offset of glucose-lowering effect for faster aspart versus insulin aspart in subjects with type 1 diabetes mellitus with different anti-insulin aspart antibody levels

\begin{tabular}{|c|c|c|c|c|c|c|c|c|c|}
\hline \multirow{2}{*}{$\begin{array}{l}\text { Anti-IAsp } \\
\text { antibody } \\
\text { quartiles }\end{array}$} & \multicolumn{3}{|c|}{$\begin{array}{l}\text { Anti-IAsp antibody level at baseline } \\
(\% \mathrm{~B} / \mathrm{T})\end{array}$} & \multicolumn{3}{|c|}{$\mathrm{AUC}_{\mathrm{GIR}, 0-60 \min }(\mathrm{mg} / \mathrm{kg})$} & \multicolumn{3}{|c|}{$\mathrm{AUC}_{\mathrm{GIR}, 2-t}(\mathrm{mg} / \mathrm{kg})$} \\
\hline & $\begin{array}{l}\text { Arithmetic } \\
\text { mean }\end{array}$ & Minimum & Maximum & $\begin{array}{l}\text { Faster } \\
\text { aspart } \\
\text { (geometric } \\
\text { mean) }\end{array}$ & $\begin{array}{l}\text { IAsp } \\
\text { (geometric } \\
\text { mean) }\end{array}$ & $\begin{array}{l}\text { Geometric } \\
\text { mean treat- } \\
\text { ment ratio } \\
\text { (faster aspart/ } \\
\text { IAsp) }\end{array}$ & $\begin{array}{l}\text { Faster } \\
\text { aspart } \\
\text { (geometric } \\
\text { mean) }\end{array}$ & $\begin{array}{l}\text { IAsp } \\
\text { (geometric } \\
\text { mean) }\end{array}$ & $\begin{array}{l}\text { Geometric } \\
\text { mean treatment } \\
\text { ratio (faster } \\
\text { aspart/IAsp) }\end{array}$ \\
\hline Quartile 1 & 1.65 & -0.11 & 3.11 & 149.5 & 112.3 & 1.33 & 621.0 & 738.4 & 0.84 \\
\hline Quartile 2 & 4.86 & 3.12 & 6.53 & 162.9 & 112.9 & 1.44 & 676.2 & 744.7 & 0.91 \\
\hline Quartile 3 & 10.46 & 6.65 & 15.68 & 149.0 & 119.0 & 1.25 & 746.2 & 815.3 & 0.92 \\
\hline Quartile 4 & 26.94 & 15.84 & 66.94 & 165.8 & 122.9 & 1.35 & 718.2 & 775.4 & 0.93 \\
\hline
\end{tabular}

Results are based on 112 subjects

$A U C_{G I R, 0-60 \mathrm{~min}}$ area under the curve for the glucose infusion rate from time zero to $60 \mathrm{~min}, A U C_{G I R, 2-t}$ area under the curve for the glucose infusion rate from $2 \mathrm{~h}, I A s p$ insulin aspart, $\% B / T$ percent bound/total

(a)

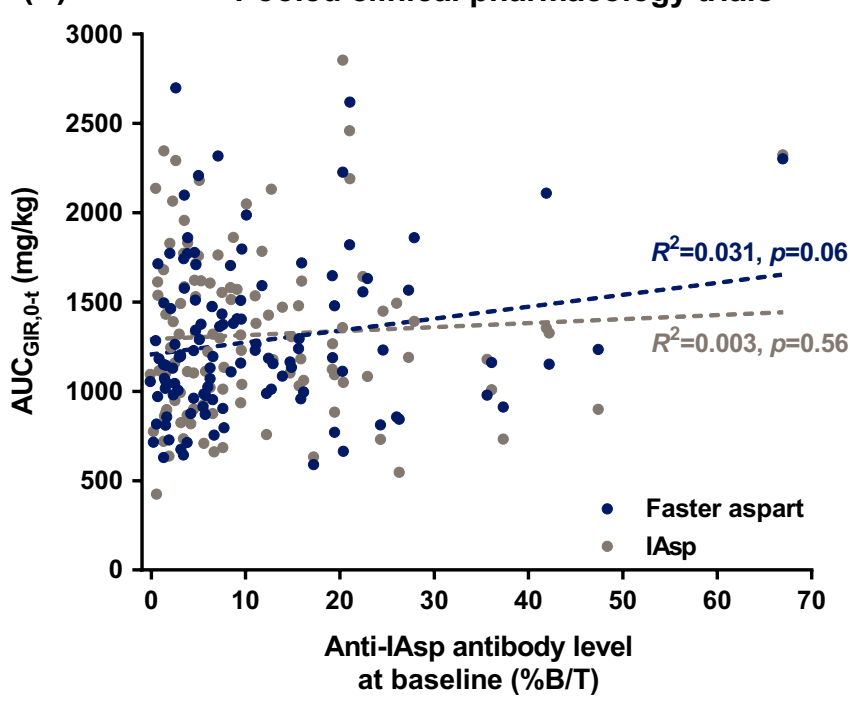

(b)

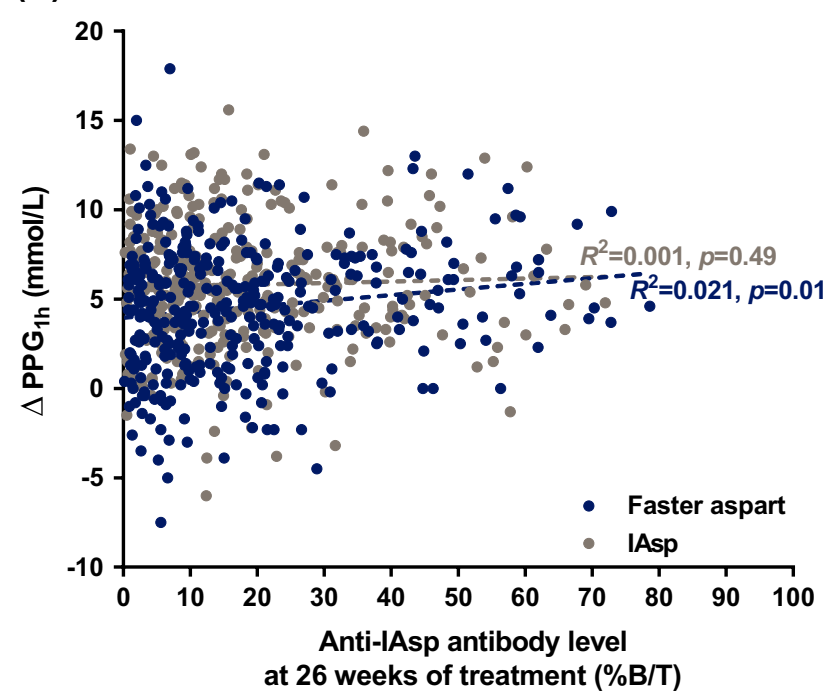

for faster aspart (blue) and IAsp (grey), respectively, including the coefficients of determination $\left(R^{2}\right)$ and the $p$ values for a test of the slope being equal to zero (i.e. horizontal regression line). $A U C_{G I R, 0-t}$ total area under the curve for glucose infusion rate, IAsp insulin aspart, $\% B / T$ percent bound/total, $\Delta P P G_{l h} 1$-h postprandial plasma glucose increment
Fig. 3 Relationship between anti-IAsp antibody level and total glucose-lowering effect $\left(\mathrm{AUC}_{\mathrm{GIR}, 0-t}\right)$ in three pooled clinical pharmacology trials (a) or $\Delta \mathrm{PPG}_{1 \mathrm{~h}}$ in a phase IIIa trial (b) with faster aspart and IAsp. Results are based on 112 subjects in the pooled clinical pharmacology trials and 344 (faster aspart) and 348 (IAsp) subjects in the phase IIIa trial. Dotted lines are the estimated regression lines 
and insulin-like growth factor-1 all circulate in the bloodstream bound to thyroid-binding globulin, sex hormonebinding globulin, growth hormone-binding protein and insulin-like growth factor-binding protein, respectively, in addition to their free fractions [25]. For all these hormones as well as for insulin, it is hypothesised that their effects are proportional to the free hormone concentration in contrast to the total hormone concentration $[1,2,25]$. This is important to bear in mind when assaying these hormones with the purpose of relating their pharmacokinetic properties to pharmacodynamic or effect measures.

A number of older studies have demonstrated altered insulin pharmacokinetics in subjects with higher versus lower anti-insulin antibody levels. The rise in insulin concentration shortly after dosing was delayed and the time to maximum concentration and the half-life were both extended in subjects with higher anti-insulin antibody levels $[3,26]$. However, the clinical significance of these findings is unclear since several later studies have shown no effect of anti-insulin antibodies on the pharmacodynamic properties of insulin products $[23,27,28]$. In accordance with the latter, the present study showed that early glucose-lowering effect within $60 \mathrm{~min}$ of dosing as well as late glucoselowering effect from $2 \mathrm{~h}$ after dosing did not consistently change across the four quartiles of anti-IAsp antibody levels. Furthermore, total glucose-lowering effect appeared to be essentially unaffected by the level of anti-IAsp antibodies. Importantly, studies have shown that anti-insulin antibodies have no clinically significant impact on the efficacy or safety of various insulin products including inhaled insulin, which is known to elicit a greater anti-insulin antibody response than seen after subcutaneous insulin administration $[4,27,29-31]$. Thus, it seems reasonable to at least conclude that development of low-to-moderate levels of anti-insulin antibodies are of limited clinical relevance for patients with diabetes.

It has previously been shown that offset of exposure occurs earlier for faster aspart than for IAsp when pharmacokinetics were measured as free IAsp [16]. In the current study, this finding was confirmed also when measuring total IAsp. A potential benefit of the earlier offset of faster aspart versus IAsp could be an improved match between meal glucose absorption and insulin exposure and effect in the late postprandial period. This, in turn, could reduce the risk of late postprandial hypoglycaemia resulting from an insulin effect that lasts longer than it takes to fully absorb glucose following a meal [32]. Indeed, it was recently shown in an analysis of two phase III trials comparing faster aspart and IAsp in a basal-bolus regimen in subjects with T1DM that the rate of meal-related severe or blood glucose-confirmed hypoglycaemia in the period from 3 to $4 \mathrm{~h}$ post-meal was significantly lower for faster aspart than for IAsp in one of the trials [33]. The same analysis also showed a significantly higher rate of hypoglycaemia for faster aspart than for IAsp in the period from 0 to $1 \mathrm{~h}$ post-meal in the other trial. These findings suggest that treatment differences between faster aspart and IAsp in the meal-related rate of hypoglycaemia may reflect the faster onset and offset of glucose-lowering effect for faster aspart than for IAsp [33].

Strengths of the current investigation included the high number of subjects in the analyses, the wide span of demographics among the analysed population and the comparable designs of the clinical pharmacology trials, particularly regarding pharmacokinetic methodology and choice of comparator. It might be a limitation of the pooled analysis of clinical pharmacology trials that only a few subjects with moderate-to-high anti-IAsp antibody titres were included. In order to address this limitation, the effect of the antiIAsp antibody level on the ratio of free/total IAsp for AUC IAsp, $0-t$ was investigated in a much larger group of subjects from a phase IIIa trial with faster aspart and IAsp. Based on the same phase IIIa trial, the relationship between anti-IAsp antibody levels and 1-h postprandial glucose increment during a meal test was investigated. In the phase IIIa trial, maximum observed anti-IAsp antibody titres were higher than in the clinical pharmacology trials (ESM Online Resource 1, Table S3) and a considerably greater number of subjects had moderate-to-high anti-IAsp antibody levels (Figs. 2, 3). Both analyses confirmed the conclusions from the pooled clinical pharmacology analysis. Notably, the slightly increasing slopes of the estimated regression lines for faster aspart in Fig. 3 indicate that, based on the clinical pharmacology trials, higher anti-IAsp antibody level would be associated with slightly greater glucose-lowering effect and, based on the phase IIIa trial, a higher anti-IAsp antibody level would be associated with slightly less glucose-lowering effect. Based on these findings combined with the very low coefficients of determination $\left(R^{2}\right)$, it should be reasonable to conclude that higher anti-IAsp antibody levels do not influence faster aspart or IAsp pharmacodynamics.

Another limitation of the present investigation was that it could not be directly estimated whether free or total IAsp is the best predictor of glucose-lowering effect. In the clinical pharmacology trials, each subject received only one dose level, and in the majority of trials this dose level was the same for all subjects $(0.2 \mathrm{U} / \mathrm{kg})$. Therefore, the range of exposure values within the population was too narrow to reliably investigate the pharmacokinetic/pharmacodynamic relationship. In the literature, the consensus is that circulating free insulin represents the biologically active pool and thus may be the best predictor of glucose-lowering effect $[1,2]$. 


\section{Conclusion}

In a pooled analysis of four clinical pharmacology trials in subjects with T1DM, faster onset of appearance, greater early exposure and earlier offset of exposure were shown with faster aspart than with IAsp, both when using free and total IAsp to assess pharmacokinetics. Higher anti-IAsp antibody levels were associated with greater exposure for total than for free IAsp. However, the level of anti-IAsp antibodies did not appear to affect the overall glucose-lowering effect of faster aspart and IAsp or the greater early glucoselowering effect and the smaller late glucose-lowering effect provided by faster aspart than by IAsp.

Acknowledgements The authors would like to thank Carsten Roepstorff, PhD, CR Pharma Consult, Copenhagen, Denmark for providing medical writing support, which was funded by Novo Nordisk.

\section{Compliance with Ethical Standards}

Ethical approval and informed consent All procedures performed in studies involving human participants were in accordance with the ethical standards of the institutional and/or national research committee and with the 1964 Helsinki Declaration and its later amendments or comparable ethical standards. Informed consent was obtained from all individual participants included in the study.

Funding This investigation was funded by Novo Nordisk.

Conflict of interest Hanne Haahr and Lars Erichsen are employees and shareholders of Novo Nordisk. Theis Gondolf is an employee of Novo Nordisk. Thomas R. Pieber has received research support from AstraZeneca and Novo Nordisk, has served in advisory panels for AstraZeneca, Bristol-Myers Squibb, Eli Lilly, Novo Nordisk and Roche Diabetes Care, and is an employee of CBmed - Center for Biomarker Research in Medicine (a publically funded research centre). Chantal Mathieu serves or has served on advisory panels for Novo Nordisk, Sanofi, Merck Sharp and Dohme, Eli Lilly, Novartis, Bristol-Myers Squibb, AstraZeneca, Pfizer, Janssen Pharmaceuticals, Boehringer Ingelheim, Hanmi Pharmaceuticals, Roche Diagnostics, Medtronic, Mannkind, Intrexon, Dianax and UCB, and as a speaker for Novo Nordisk, Sanofi, Merck Sharp and Dohme, Eli Lilly, Boehringer Ingelheim, AstraZeneca and Novartis. Financial compensation for these activities has been received by KU Leuven. KU Leuven has received research support for Chantal Mathieu from Medtronic, Novo Nordisk, Sanofi, Merck Sharp and Dohme, Eli Lilly, Roche Diagnostics, Abbott, Intrexon and Novartis. Tim Heise is a shareholder of Profil, which has received research funds from Adocia, Boehringer Ingelheim, Dance Pharmaceuticals, Eli Lilly, Johnson \& Johnson, MedImmune, Merck Sharp and Dohme, Mylan, Nordic Bioscience, Novo Nordisk, Poxel, Roche Diagnostics, Saniona, Sanofi, Senseonics and Zealand Pharma. In addition, Tim Heise is member of advisory panels for Novo Nordisk and Mylan and received speaker honoraria and travel grants from Dexcom, Eli Lilly, Mylan, Novo Nordisk, Sanofi and Zealand Pharma. Masanari Shiramoto has no conflicts of interest to declare.

Data availability Will individual participant data be available (including data dictionaries)? Individual participant data will be shared in datasets in a de-identified/anonymised format. What data in particular will be shared? Datasets from Novo Nordisk-sponsored clinical research completed after 2001 for product indications approved in both the EU and USA. What other documents will be available? The study protocol and redacted Clinical Study Report (CSR) will be available according to Novo Nordisk data sharing commitments. When will data be available (start and end dates)? The data will be available permanently after research completion and approval of product and product use in both the EU and USA. There is no end date. With whom will data be shared? With bona fide researchers submitting a research proposal requesting access to data. For what types of analyses? For use as approved by the Independent Review Board (IRB) according to the IRB Charter (see novonordisk-trials.com). By what mechanism will data be made available? Access request proposal form and the access criteria can be found at novonordisk-trials.com. The data will be made available on a specialised $\mathrm{SAS}^{\circledR}$ data platform.

Open Access This article is distributed under the terms of the Creative Commons Attribution-NonCommercial 4.0 International License (http://creativecommons.org/licenses/by-nc/4.0/), which permits any noncommercial use, distribution, and reproduction in any medium, provided you give appropriate credit to the original author(s) and the source, provide a link to the Creative Commons license, and indicate if changes were made.

\section{References}

1. Arnqvist H, Olsson PO, von Schenck H. Free and total insulin as determined after precipitation with polyethylene glycol: analytical characteristics and effects of sample handling and storage. Clin Chem. 1987;33:93-6.

2. Sapin R. Insulin assays: previously known and new analytical features. Clin Lab. 2003;49:113-21.

3. Van Haeften TW. Clinical significance of insulin antibodies in insulin-treated diabetic patients. Diabetes Care. 1989;12:641-8.

4. Fineberg SE, Kawabata TT, Finco-Kent D, Fountaine RJ, Finch GL, Krasner AS. Immunological responses to exogenous insulin. Endocr Rev. 2007;28:625-52.

5. Klingensmith GJ. Insulin antibodies-are they still with us? Do they matter? Pediatr Diabetes. 2011;12:75-7.

6. Sapin R. Anti-insulin antibodies in insulin immunometric assays: a still possible pitfall. Eur J Clin Chem Clin Biochem. 1997;35:365-7.

7. Biester T, Danne T, Bläsig S, et al. Pharmacokinetic and prandial pharmacodynamic properties of insulin degludec/insulin aspart in children, adolescents, and adults with type 1 diabetes. Pediatr Diabetes. 2016;17:642-9.

8. Lamos EM, Younk LM, Tate DB, Davis SN. Pharmacokinetics and pharmacodynamics of insulin glargine-insulin glulisine basalbolus and twice-daily premixed analog insulin in type 1 diabetes mellitus patients during three standardized meals. J Clin Transl Endocrinol. 2015;3:14-20.

9. Danne T, Becker RH, Heise T, Bittner C, Frick AD, Rave K. Pharmacokinetics, prandial glucose control, and safety of insulin glulisine in children and adolescents with type 1 diabetes. Diabetes Care. 2005;28:2100-5.

10. de la Peña A, Seger M, Soon D, et al. Bioequivalence and comparative pharmacodynamics of insulin lispro $200 \mathrm{U} / \mathrm{mL}$ relative to insulin lispro (Humalog ${ }^{\circledR}$ ) $100 \mathrm{U} / \mathrm{mL}$. Clin Pharmacol Drug Dev. 2016;5:69-75.

11. FDA. Novolog: prescribing information. 2017. https://www.acces sdata.fda.gov/drugsatfda_docs/label/2017/020986Orig 1s079 Lbledt.pdf. Accessed 19 Sep 2018.

12. EMA. Fiasp: summary of product characteristics. 2017. http://www.ema.europa.eu/docs/en_GB/document_library/ 
EPAR_-_Product_Information/human/004046/WC50022089 0.pdf. Accessed 19 Sep 2018.

13. FDA. Fiasp: prescribing information. 2018. https://www.acces sdata.fda.gov/drugsatfda_docs/label/2018/208751s001lbl.pdf. Accessed 19 Sep 2018.

14. Buckley ST, Kildegaard J, Høiberg-Nielsen R, et al. Mechanistic analysis into the mode(s) of action of niacinamide in faster-acting insulin aspart. Diabetes Technol Ther. 2016;18(Suppl 1):A116-7.

15. Heise T, Hövelmann U, Brøndsted L, et al. Faster-acting insulin aspart: earlier onset of appearance and greater early pharmacokinetic and pharmacodynamic effects than insulin aspart. Diabetes Obes Metab. 2015;17:682-8.

16. Heise T, Pieber TR, Danne T, Erichsen L, Haahr H. A pooled analysis of clinical pharmacology trials investigating the pharmacokinetic and pharmacodynamic characteristics of fast-acting insulin aspart in adults with type 1 diabetes. Clin Pharmacokinet. 2017;56:551-9.

17. Heise T, Hövelmann U, Zijlstra E, et al. A comparison of pharmacokinetic and pharmacodynamic properties between faster-acting insulin aspart and insulin aspart in elderly subjects with type 1 diabetes mellitus. Drugs Aging. 2017;34:29-38.

18. Shiramoto M, Nishida T, Hansen AK, Haahr H. Fast-acting insulin aspart in Japanese patients with type 1 diabetes: faster onset, higher early exposure and greater early glucose-lowering effect relative to insulin aspart. J Diabetes Investig. 2018;9:303-10.

19. Basu A, Pieber TR, Hansen AK, et al. Greater early postprandial suppression of endogenous glucose production and higher initial glucose disappearance is achieved with fast-acting insulin aspart compared with insulin aspart. Diabetes Obes Metab. 2018;20:1615-22.

20. Russell-Jones D, Bode BW, De Block C, et al. Fast-acting insulin aspart improves glycemic control in basal-bolus treatment for type 1 diabetes: results of a 26 -week multicenter, active-controlled, treat-to-target, randomized, parallel-group trial (Onset 1). Diabetes Care. 2017;40:943-50.

21. Kurtz AB, Mustaffa BE, Daggett PR, Nabarro JD. Effect of insulin antibodies on free and total plasma-insulin. Lancet. 1977;2:56-8.

22. Waldhäusl WK, Bratusch-Marrain P, Kruse V, Jensen I, Nowotny $\mathrm{P}$, Vierhapper H. Effect of insulin antibodies on insulin pharmacokinetics and glucose utilization in insulin-dependent diabetic patients. Diabetes. 1985;34:166-73.
23. Hübinger A, Becker A, Gries FA. Total insulin levels in type 1 diabetic patients with insulin antibodies and their effect on insulin requirement and metabolic control. Diabetes Res. 1988;7:65-9.

24. FDA. Humalog: prescribing information. 2017. https://www. accessdata.fda.gov/drugsatfda_docs/label/2017/020563s176lbl. pdf. Accessed 19 Sep 2018.

25. Faix JD. Principles and pitfalls of free hormone measurements. Best Pract Res Clin Endocrinol Metab. 2013;27:631-45.

26. Van Haeften TW, Bolli GB, Dimitriadis GD, Gottesman IS, Horwitz DL, Gerich JE. Effect of insulin antibodies and their kinetic characteristics on plasma free insulin dynamics in patients with diabetes mellitus. Metabolism. 1986;35:649-56.

27. Heise T, Bott $S$, Tusek $C$, et al. The effect of insulin antibodies on the metabolic action of inhaled and subcutaneous insulin: a prospective randomized pharmacodynamic study. Diabetes Care. 2005;28:2161-9.

28. Chen JW, Frystyk J, Lauritzen T, Christiansen JS. Impact of insulin antibodies on insulin aspart pharmacokinetics and pharmacodynamics after 12-week treatment with multiple daily injections of biphasic insulin aspart 30 in patients with type 1 diabetes. Eur J Endocrinol. 2005; 153:907-13.

29. Lindholm A, Jensen LB, Home PD, Raskin P, Boehm BO, Råstam J. Immune responses to insulin aspart and biphasic insulin aspart in people with type 1 and type 2 diabetes. Diabetes Care. 2002;25:876-82.

30. Hermansen K, Rönnemaa T, Petersen AH, Bellaire S, Adamson U. Intensive therapy with inhaled insulin via the AERx insulin diabetes management system: a 12-week proof-of-concept trial in patients with type 2 diabetes. Diabetes Care. 2004;27:162-7.

31. Fineberg SE, Kawabata TT, Krasner AS, Fineberg NS. Insulin antibodies with pulmonary delivery of insulin. Diabetes Technol Ther. 2007;9:S102-10.

32. Heinemann L, Muchmore DB. Ultrafast-acting insulins: state of the art. J Diabetes Sci Technol. 2012;6:728-42.

33. De Block C, Carlson A, Rose L, Gondolf T, Gorst-Rasmussen A, Lane W. Hypoglycemia with mealtime fast-acting insulin aspart vs. insulin aspart across two large type 1 diabetes trials [poster no. 96-LB]. American Diabetes Association, 78th Scientific Sessions; 22-26 Jun 2018; Orlando. 